\title{
INTEGRAL and Swift observations of EXO 2030+375 during a giant outburst ${ }^{\star}$
}

\author{
D. Klochkov ${ }^{1}$, D. Horns ${ }^{1}$, A. Santangelo ${ }^{1}$, R. Staubert ${ }^{1}$, A. Segreto ${ }^{3}$, C. Ferrigno ${ }^{3}$, P. Kretschmar ${ }^{4}$, I. Kreykenbohm ${ }^{1,5}$, \\ A. La Barbera ${ }^{3}$, N. Masetti ${ }^{8}$, M. McCollough ${ }^{2}$, K. Pottschmidt ${ }^{6}$, G. Schönherr ${ }^{1}$, and J. Wilms ${ }^{7}$ \\ 1 Institut für Astronomie und Astrophysik, University of Tübingen, Sand 1, 72076 Tübingen, Germany \\ e-mail: klochkov@astro.uni-tuebingen.de \\ 2 Smithsonian Astrophysical Observatory, 60 Garden Street, Cambridge, MA 02138, USA \\ 3 INAF IFC-Pa, via U. La Malfa 153, 90146 Palermo, Italy \\ 4 Integral SOC ESA, Madrid, Spain \\ 5 INTEGRAL Science Data Centre, Chemin d'Écogia, 16, 1290, Versoix, Switzerland \\ 6 Center for Astrophysics and Space Sciences, University of California, San Diego, La Jolla, CA 92093-0424, USA \\ 7 Dr. Karl Remeis-Sternwarte, Astronomisches Institut, Universität Erlangen-Nürnberg, Sternwartstr. 7, 96049 Bamberg, Germany \\ 8 INAF IASF-Bo, via Gobetti 101, 40129 Bologna, Italy
}

Received 23 November 2006 / Accepted 26 January 2007

\section{ABSTRACT}

\begin{abstract}
Aims. We investigate the X-ray spectral and timing properties of the high mass X-ray binary EXO 2030+375 observed during its June-September 2006 giant (type II) outburst.

Methods. The data analyzed in this work are from partly simultaneous observations with INTEGRAL and Swift. The pulse period $P$ and its temporal derivative $\dot{P}$ are measured. X-ray pulse profiles in different energy ranges and time intervals are constructed. Pulse averaged X-ray spectra for different time intervals are studied.

Results. We report a strong spin-up of the source during the outburst, comparable to that observed in 1985 during the previous giant outburst when the source was discovered. The value of $\dot{P}$ is found to be linearly related to the X-ray luminosity of the source during the outburst. For the first time the hard X-ray $(>25 \mathrm{keV})$ characteristics of the source during a type II outburst are studied. The X-ray pulse profiles apparently change with luminosity. The X-ray spectral continuum in the $3-120 \mathrm{keV}$ energy range is modeled with an absorbed power law with an exponential cutoff around $E \sim 26 \mathrm{keV}$. An iron emission line at $\sim 6-7 \mathrm{keV}$ is observed. The spectrum reveals some features between 10 and $20 \mathrm{keV}$ which can be modeled either by a broad emission line at $\sim 13-15 \mathrm{keV}$ (a "bump") or by two absorption lines at $\sim 10$ and $\sim 20 \mathrm{keV}$.
\end{abstract}

Key words. X-ray binaries - stars: neutron - accretion, accretion disks

\section{Introduction}

Discovered by EXOSAT in 1985 during a giant outburst (Parmar et al. 1989), the transient accreting pulsar EXO 2030+375 belongs to a high mass X-ray binary system with a B0 Ve star as optical companion (Coe et al. 1988). The orbital period and eccentricity are $\sim 46 \mathrm{~d}$ and $\sim 0.42$, respectively (Wilson et al. 2005). According to the generally accepted model for Be/X-ray binaries, the X-ray emission in such systems during outbursts is produced when the compact object accretes from a quasi-Keplerian disk around the equator of the rapidly rotating Be star. Such a mechanism explains normal (type I) outbursts with X-ray luminosity $L_{\mathrm{X}} \lesssim 10^{37} \mathrm{erg} \mathrm{s}^{-1}$, associated with the periastron passage of the compact object. Sometimes, however, Be/X-ray binaries show giant (or type II) outbursts with X-ray luminosity $L_{\mathrm{X}} \gtrsim 10^{37} \mathrm{erg} \mathrm{s}^{-1}$. Such outbursts are thought to be due to a dramatic expansion of the disk surrounding the Be star, leading to the formation of an accretion disk around the compact object (Coe 2000).

* Based on observations with INTEGRAL, an ESA project with instruments and science data centre funded by ESA member states (especially the PI countries: Denmark, France, Germany, Italy, Switzerland, Spain), Czech Republic and Poland, and with the participation of Russia and the USA.
During the 1985 giant outburst the X-ray luminosity of the source reached a value of $L_{1-20 \mathrm{keV}} \sim 2 \times 10^{38} \mathrm{erg} \mathrm{s}^{-1}$ (for a distance of $7.1 \mathrm{kpc}$, Wilson et al. 2002). The spin period of the pulsar ( 42s) changed dramatically, with a spin-up time scale $-P / \dot{P} \approx 30$ yr (Parmar et al. 1989).

From the nearly continuous monitoring of the source with the Burst and Transient Source Experiment (BATSE) on the Compton Gamma Ray Observatory (CGRO) and the All Sky Monitor $(A S M)$ on the Rossi X-ray Timing Explorer (RXTE) it is known that the pulse period remained roughly constant for about a year after the beginning of the monitoring in 1991, followed by 2 years of relatively slow spin-up and 6 years of slow spin-down (Wilson et al. 2002, 2005). After 2002 a transition to a global spin-up along with an overall brightening of the normal outbursts was reported by Wilson et al. (2005).

The giant outburst of EXO 2030+375 presented here (Corbet \& Levine 2006; Krimm et al. 2006; McCollough et al. 2006) is the second of its kind since the discovery of the source. The X-ray luminosity at the maximum of the outburst reached $L_{1-20 \mathrm{keV}} \sim 1.2 \times 10^{38} \mathrm{erg} \mathrm{s}^{-1}$ (Wilson \& Finger 2006). In this Letter, based on INTEGRAL and Swift data, we investigate in Sect. 2.1 the pulse period and the spin-up rate of the source and in Sect. 2.2 we construct X-ray pulse profiles for different energy ranges and time intervals. To characterize the broad-band 
Table 1. Summary of observations.

\begin{tabular}{ccccc}
\hline \hline Obs. & Instrument & $\begin{array}{c}\text { Obs. time } \\
(\mathrm{ks})\end{array}$ & $\begin{array}{c}\text { Mean } \\
\text { MJD }\end{array}$ & $\begin{array}{c}\text { Mean ASM } \\
\text { flux (mCrab) }\end{array}$ \\
\hline OBS1 & ISGRI+JEM-X & 62 & 53942.9 & 500 \\
OBS2 & ISGRI+JEM-X & 140 & 53967.6 & 500 \\
OBS2 & XRT + BAT & 6.3 & 53967.3 & 500 \\
OBS3 & XRT + BAT & 6.4 & 54002.5 & 160 \\
\hline
\end{tabular}

Table 2. Pulse period development. The uncertainties in parentheses (68\%) refer to the last digit(s).

\begin{tabular}{ccccc}
\hline \hline Obs. & MJD & $\begin{array}{c}P \\
{[\mathrm{~s}]}\end{array}$ & $\begin{array}{c}-\mathrm{d} P / \mathrm{d} t \\
{\left[10^{-8} \mathrm{~s} / \mathrm{s}\right]}\end{array}$ & $\begin{array}{c}\text { Flux (3-10 keV) } \\
{\left[10^{-9} \mathrm{erg} \mathrm{cm}^{-2} \mathrm{~s}^{-1}\right]}\end{array}$ \\
\hline OBS1 & 53942.7714 & $41.57958(2)$ & $2.93(12)$ & $9.61(6)$ \\
OBS2 & 53967.4260 & $41.51706(5)$ & $3.29(19)$ & $10.41(4)$ \\
OBS3 & 54002.3877 & $41.45954(4)$ & $0.67(10)$ & $3.25(3)$ \\
\hline
\end{tabular}

spectral behaviour we perform in Sect. 2.3 the spectral analysis of pulse-phase averaged spectra of the source. A detailed study of the timing and spectral behaviour as a function of the luminosity is beyond the scope of this Letter and will be presented in a forthcoming paper.

\section{Observations and data analysis}

EXO 2030+375 was observed by INTEGRAL (Winkler et al. 2003) on 27 July and 19-21 August 2006 and by Swift (Gehrels et al. 2004) on 19-20 August and 23-25 September 2006. Another simultaneous observation by INTEGRAL and Swift was performed on 6-8 October 2006, but the data were not available at the time of writing this Letter and will be presented later. For our analysis we used the data obtained with the instruments IBIS/ISGRI (20-300 keV, Ubertini et al. 2003) and JEM-X (3-30 keV, Lund et al. 2003) onboard INTEGRAL as well as XRT (0.2-10 keV, Burrows et al. 2005) and BAT (15-150 keV, Barthelmy et al. 2005) of Swift. For the analysis of ISGRI and $J E M-X$ data the Off-line Science Analysis (OSA) software (version 5.1) was used (Courvoisier et al. 2003). Analysis of $X R T$ data was done with the standard HEADAS package (version 6.0.4).

Table 1 contains the summary of the observations analyzed in this work. A part of the ASM light curve ${ }^{1}$ of the source including three normal outbursts and the 2006 giant outburst is presented in Fig. 1. The observations analyzed in this paper are marked by solid lines and consist of three sets of pointings. The first set was done by INTEGRAL. The second set contains simultaneous observations by Swift and INTEGRAL. The last set of observations was performed by Swift. These three sets will be referred to as OBS1, OBS2, and OBS3 throughout the paper. OBS1 and OBS2 observations were made when the source was at approximately the same luminosity level, before and after the maximum of the outburst. OBS3 set was made during the decay of the outburst when the luminosity of the source dropped by a factor of $\sim 3$.

\subsection{Pulse period behaviour}

For the timing analysis all times were translated to the solar system barycenter and corrected for orbital motion in the binary.

1 We used the results provided by the ASM/RXTE teams at MIT and at the RXTE SOF and GOF at NASA's GSFC.

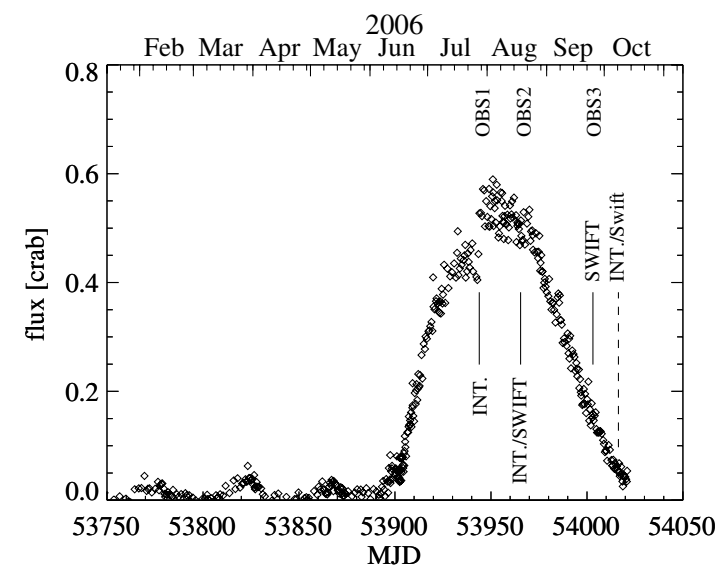

Fig. 1. The ASM light curve of EXO 2030+375. The times of INTEGRAL (INT.) and Swift observations analyzed in this work are marked by solid lines. The dashed line shows another simultaneous observation by INTEGRAL and Swift for which the data are not yet available.

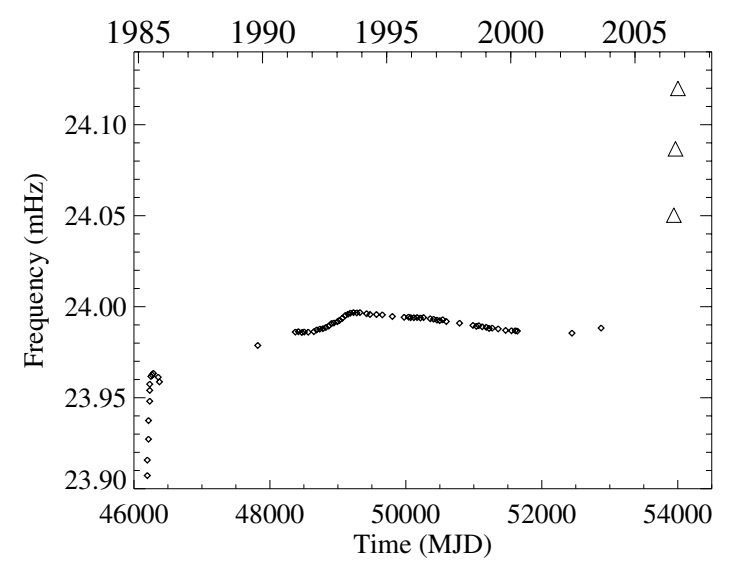

Fig. 2. Spin frequency of EXO 2030+375. Small diamonds show the spin frequency development before the 2006 giant outburst (they are taken from Wilson et al. 2002, 2005). The large triangles correspond to our pulse frequency measurements during the outburst. The uncertainties of measurements and duration of each observation are smaller than the size of symbols in the plot.

The pulse periods and associated derivatives were determined for each of the three observation periods individually (Table 2). These values were found by employing initial epoch-folding and a subsequent phase connection analysis similar to Ferrigno et al. (2007) using well defined pulse profiles from a sufficiently large number of pulses. Variation in pulse shape inside each observation is marginal and does not affect our method.

Figure 2 displays our points together with the historical pulse period development as presented by Wilson et al. $(2002,2005)$. The three datasets are consistent with a global solution (valid between MJD 53943 and MJD 54003) with the following parameters: $P=41.47268(6) \mathrm{s}, \dot{P}=-1.627(5) \times 10^{-8} \mathrm{~s} / \mathrm{s}$ and $\ddot{P}=$ $4.058(25) \times 10^{-15} \mathrm{~s}^{-1}$ for the reference MJD 53992.25 (TDB). The uncertainties in parentheses $(68 \%)$ refer to the last digit(s). The characteristic spin-up time scale, $-P / \dot{P}$, is $\sim 40$ years, somewhat longer than during the first giant outburst. We also note that the $\dot{P}$ appears to be linearly correlated with the X-ray luminosity (see last two columns in Table 2). The formal correlation coefficient is 1.0 and the slope from a linear fit is $36 \pm 2\left(\mathrm{~s} \mathrm{~s}^{-1}\right) /\left(\mathrm{erg} \mathrm{cm}^{-2} \mathrm{~s}^{-1}\right)$, although it is based on three data points. 


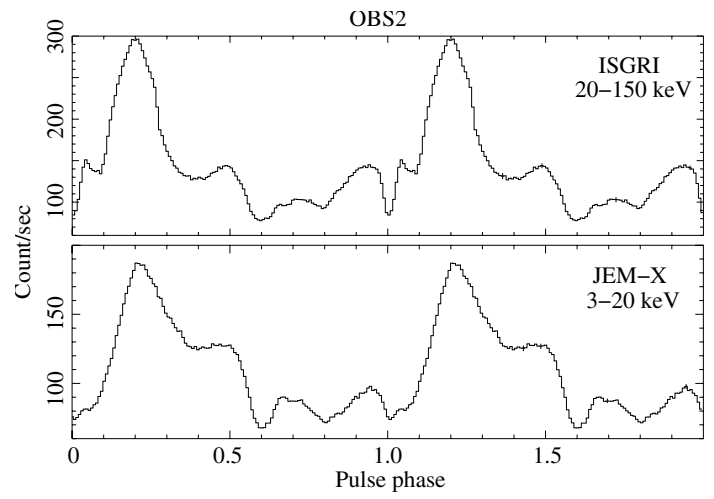

Fig. 3. X-ray pulse profiles obtained with ISGRI (top panel) and JEM-X (bottom panel) during OBS2 (MJD 53966), near the maximum of the outburst. Phase 0.0 was arbitrarily chosen to be defined by the dip-like feature before the main peak. Typical error bars are indicated.

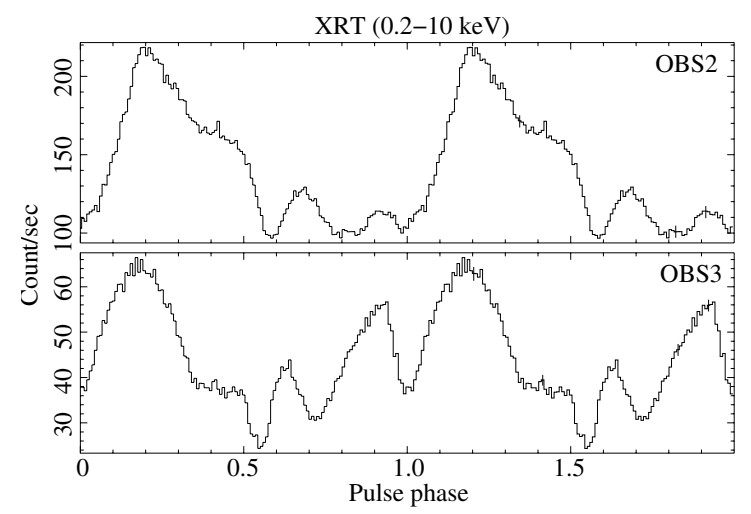

Fig. 4. X-ray pulse profile obtained with XRT during OBS2 (top panel) and during OBS3 (bottom panel). Typical error bars are indicated.

\subsection{Pulse profiles}

Using the values of $P$ and $\dot{P}$ determined here (Table 2) we constructed pulse profiles for each observation. Pulse profiles from OBS1 and OBS2 are found to be very similar. This can be expected considering that the luminosity of the source during these observations was approximately the same. The results are shown in Figs. 3 and 4. We do not show pulse profiles from OBS1 since there are similar to OBS2.

Figure 4 shows the $0.2-10 \mathrm{keV}$ pulse profiles obtained with $X R T$. One can see that the pulse profile obtained in OBS3, during the decay of the outburst, appears different from that obtained in OBS2 when the luminosity was higher.

The analysis of the pulse profiles, including the comparison with those obtained during the 1985 outburst and during normal outbursts, is on-going.

\subsection{Spectral analysis}

For OBS1 pulse averaged X-ray spectra of ISGRI and JEM-X were analyzed. For OBS2 spectra from ISGRI, JEM-X, XRT and $B A T$ were fitted simultaneously. In order to account for small scale uncertainties in the response matrices of the respective instruments systematic uncertainties were added at a level of $1 \%$ for $I S G R I$ and $3 \%$ for $J E M-X$ and XRT. For BAT we used energydependent systematic errors provided by the $H E A D A S$ package. We note that there are additional global systematic uncertainties with the instruments on INTEGRAL (e.g. the canonical spectrum
Table 3. Best fit spectral parameters of EXO 2030+375 using the model with a "bump" around $15 \mathrm{keV}$ (see text). $1 \sigma(68 \%)$-uncertainties for one parameter of interest are shown.

\begin{tabular}{lcc}
\hline \hline & $53943(\mathrm{OBS} 1)$ & $53967(\mathrm{OBS} 2)$ \\
Time of observation & ISGRI, & ISGRI, JEM-X \\
$(\mathrm{MJD}) /$ Instruments & JEM-X & XRT, BAT \\
\hline$\Gamma$ & $1.93 \pm 0.01$ & $1.93_{-0.02}^{+0.01}$ \\
$E_{\text {cutoff }}[\mathrm{keV}]$ & $25.9_{-0.3}^{+0.2}$ & $26.4_{-0.5}^{+0.2}$ \\
$E_{\text {fold }}[\mathrm{keV}]$ & $26.1 \pm 0.2$ & $26.9 \pm 0.2$ \\
$E_{\mathrm{Fe}}[\mathrm{keV}]$ & - & $6.6 \pm 0.1$ \\
$\sigma_{\mathrm{Fe}}[\mathrm{keV}]$ & - & $1.2 \pm 0.1$ \\
$E_{\text {bump }}[\mathrm{keV}]$ & $15.3 \pm 0.2$ & $13.4_{-0.5}^{+0.4}$ \\
$\sigma_{\text {bump }}[\mathrm{keV}]$ & $2.7 \pm 0.2$ & $4.1_{-0.5}^{+0.3}$ \\
$N_{\mathrm{H}}\left[10^{22} \mathrm{~cm}{ }^{-2}\right]$ & $3.1 \pm 0.2$ & $3.4 \pm 0.1$ \\
$F_{\text {JEM-X }}$ & $1.0(\mathrm{fixed})$ & $1.0($ fixed $)$ \\
$F_{\text {ISGRI }}$ & $1.20 \pm 0.01$ & $1.20 \pm 0.01$ \\
$F_{\mathrm{XRT}}$ & - & $0.926 \pm 0.004$ \\
$F_{\text {BAT }}$ & - & $0.91 \pm 0.01$ \\
$\chi_{\text {red }}^{2} /$ d.o.f. & $1.1 / 259$ & $1.3 / 937$ \\
\hline
\end{tabular}

of the Crab is not reproduced). The stated uncertainties (e.g. in Tables 3 and 4) are therefore to be taken as lower limits.

To fit the broad band continuum of the source we used an absorbed power law with an exponential cutoff (XSPEC highecut model):

$\frac{\mathrm{d} N}{\mathrm{~d} E} \propto \begin{cases}E^{-\Gamma}, & \text { if } E \leq E_{\text {cutoff }} \\ E^{-\Gamma} \cdot \exp \frac{E-E_{\text {cutoff }}}{E_{\text {fold }}}, & \text { if } E>E_{\text {cutoff }}\end{cases}$

where $\mathrm{d} N / \mathrm{d} E$ is the differential count rate, $E$ is the energy of the photons; $\Gamma, E_{\text {cutoff }}$, and $E_{\text {fold }}$ are model parameters. An iron emission line at $\sim 6.5 \mathrm{keV}$ was observed during OBS2 (which has the largest total number of counts) and modeled by a Gaussian emission line. $J E M-X$ data revealed an additional feature in 10-20 keV range, which could be explained by a Gaussian emission model at $\sim 13-15 \mathrm{keV}$ (a "bump"). Alternatively, following the suggestion of a cyclotron line at $\sim 10 \mathrm{keV}$ by Wilson \& Finger (2006), we equally well fitted the spectra adding two Gaussian absorption lines at $\sim 10$ and $\sim 20 \mathrm{keV}$ to the broad band continuum. To account for large systematic uncertainties in the absolute flux measured by the instruments we introduced in our models a free multiplicative factor for each instrument: $F_{\text {ISGRI }}, F_{\mathrm{XRT}}$, and $F_{\mathrm{BAT}}$ (for JEM-X the factor was fixed to 1.0). The best fit spectral parameters for OBS1 and OBS2 are listed in Table 3 (for the model with a "bump") and Table 4 (for the model with two absorption lines). The broad band spectrum of OBS2 fitted with highecut is shown in Fig. 5a. Figures 5b-d show the residuals after fitting the spectrum by the highecut model without additional features between 10 and $20 \mathrm{keV}$, with a "bump" around $15 \mathrm{keV}$, and, alternatively, with two Gaussian absorption lines.

\section{Summary and discussion}

In June-September 2006 EXO 2030+375 has undergone the second giant (type II) outburst since its discovery in 1985. During the outburst the source has been observed with INTEGRAL and Swift. The main results of these observations can be summarized as follows:

1. The spin frequency of the pulsar has dramatically increased, indicating the presence of an accretion disk around the neutron star. The maximum value of $\dot{P}$ is found to be comparable to that observed during the first outburst during which the 
Table 4. Best fit spectral parameters of EXO 2030+375 using the model with two absorption lines at $\sim 10$ and $\sim 20 \mathrm{keV}$ (see text). $1 \sigma(68 \%)$ uncertainties for one parameter of interest are shown.

\begin{tabular}{lcc}
\hline \hline & $53943(\mathrm{OBS} 1)$ & $53967(\mathrm{OBS} 2)$ \\
Time of observation & ISGRI, & ISGRI, JEM-X \\
$(\mathrm{MJD}) /$ Instruments & JEM-X & XRT,BAT \\
\hline$\Gamma$ & $1.79 \pm 0.01$ & $1.78 \pm 0.01$ \\
$E_{\text {cutoff }}[\mathrm{keV}]$ & $19.8 \pm 0.4$ & $19.5 \pm 0.3$ \\
$E_{\text {fold }}[\mathrm{keV}]$ & $24.2 \pm 0.2$ & $24.7_{-0.2}^{+0.1}$ \\
$E_{\mathrm{Fe}}[\mathrm{keV}]$ & - & $6.51 \pm 0.03$ \\
$\sigma_{\mathrm{Fe}}[\mathrm{keV}]$ & - & $0.32_{-0.03}^{+0.04}$ \\
$E_{1 \mathrm{st} \mathrm{line}}[\mathrm{keV}]$ & $10.0_{-0.3}^{+0.2}$ & $10.6_{-0.2}^{+0.1}$ \\
$\sigma_{1 \mathrm{st} \mathrm{line}}[\mathrm{keV}]$ & $1.6_{-0.3}^{+0.4}$ & $0.7 \pm 0.2$ \\
$E_{2 \text { nd line }}[\mathrm{keV}]$ & $20.7 \pm 0.3$ & $20.6 \pm 0.3$ \\
$\sigma_{2 \text { nd line }}[\mathrm{keV}]$ & $1.3 \pm 0.3$ & $2.0_{-0.2}^{+0.3}$ \\
$N_{\mathrm{H}}\left[10^{22} \mathrm{~cm}{ }^{-2}\right]$ & $2.2 \pm 0.2$ & $3.1 \pm 0.1$ \\
$\chi_{\text {red }}^{2} / \mathrm{d} .0 . \mathrm{of}$. & $1.1 / 256$ & $1.3 / 934$ \\
$F_{\text {JEM-X }}$ & $1.0($ fixed) & $1.0($ fixed $)$ \\
$F_{\text {ISGRI }}$ & $1.25 \pm 0.01$ & $1.20 \pm 0.01$ \\
$F_{\text {XRT }}$ & - & $0.926 \pm 0.004$ \\
$F_{\text {BAT }}$ & - & $0.90 \pm 0.01$ \\
\hline
\end{tabular}

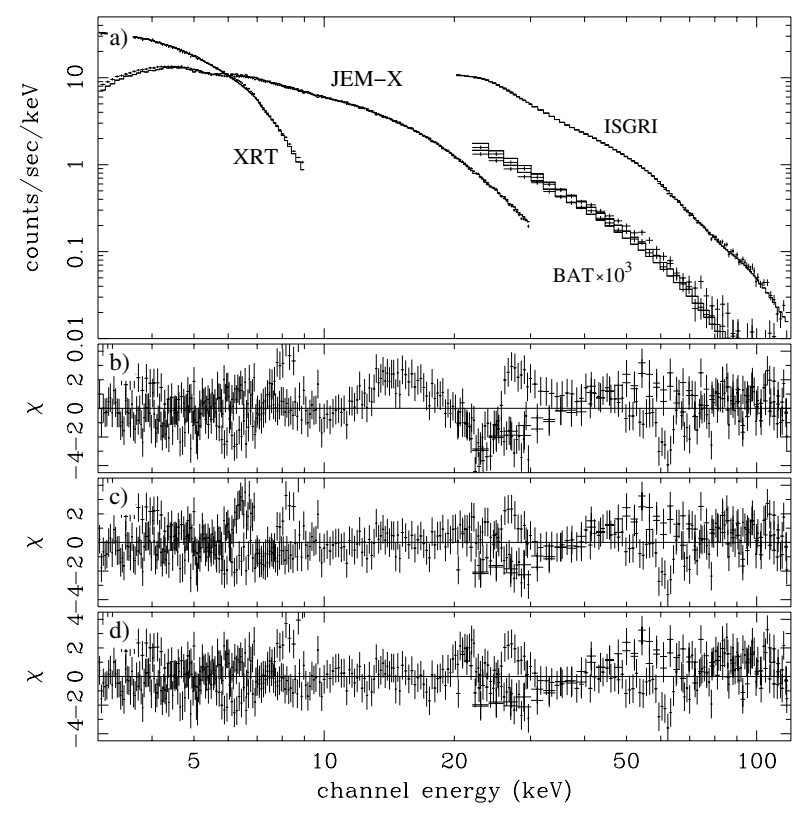

Fig. 5. The broad band spectrum of EXO 2030+375 from simultaneous fits of INTEGRAL and Swift data of OBS2 with highecut a) and residual plots after fitting it without additional features b), adding a "bump" around $15 \mathrm{keV} \mathrm{c}$ ), or alternatively including two absorption lines at $\sim 10$ and $\sim 20 \mathrm{keV}$ d).

source was discovered. The values of $\dot{P}$ determined for the three observation periods are linearly related to the X-ray luminosity, in line with previous observations (Parmar et al. 1989) and the prediction of accretion torque theory (Ghosh \& Lamb 1979).

Since the luminosity of the source and its spin-up rate could be measured, we can estimate the value of the magnetic field strength on the neutron star using the classical model of an accreting X-ray pulsar (Pringle \& Rees 1972). The equation determining the change of the angular momentum of the neutron star has the form $I \dot{\omega}=\dot{M} \sqrt{G M R_{\mathrm{A}}}$, where $I$ is the moment of inertia of the neutron star, $\omega$ is the angular frequency, $R_{\mathrm{A}}$ is the Alfvén radius, $M$ is the mass of the neutron star, and $\dot{M}$ is the accretion rate. $R_{\mathrm{A}}$ depends on the magnetic field strength and the accretion rate as $R_{\mathrm{A}} \simeq\left[\mu^{2} /(2 \dot{M} \sqrt{2 G M})\right]^{2 / 7}$, where $\mu$ is the magnetic dipole moment of the neutron star. Using the observed values of the luminosity $L$ (which can be translated into the accretion rate using the relation $\left.L \sim 0.1 \dot{M} c^{2}\right), \dot{P}$ and standard neutron star parameters, we obtain an estimate of the magnetic field strength $B \sim 1-4 \times 10^{12}$ Gauss. This would be consistent with a fundamental cyclotron line in the range $E_{\text {cyc }} \sim 10-50 \mathrm{keV}$ $\left(E_{\mathrm{cyc}}=11.6 \cdot\left(B / 10^{12} \mathrm{G}\right) \times[1+z]^{-1} \mathrm{keV}\right.$, where $z$ is the gravitational redshift which was assumed to be 0.2 in our calculations).

2. For the first time the broad band X-ray spectrum of the source (including energies above $\sim 25 \mathrm{keV}$ ) during a type II outburst is analyzed. The continuum is modeled by a power law with an exponential cutoff. An iron emission line is observed at $\sim 6-7 \mathrm{keV}$. The spectrum shows additional features between 10 and $20 \mathrm{keV}$ which can be equally well modeled with a broad emission line at $\sim 13-15 \mathrm{keV}$ or two absorption lines at $\sim 10$ and $\sim 20 \mathrm{keV}$. On the basis of phase averaged analysis we can not distinguish between the two options and we can not confirm the presence of a cyclotron line at $\sim 36 \mathrm{keV}$ reported by Reig \& Coe (1999). A preliminary pulse phase resolved analysis shows that the spectrum is highly variable with pulse phase. This means that any interpretation of the phase averaged spectrum should be made with caution. Moreover, residual features around $25 \mathrm{keV}$ may hint at not yet well understood calibration uncertainties.

3 . The shape of the profiles is found to vary during the outburst, probably related to the luminosity.

Pulse-phase-resolved analysis along with the analysis of the spectral and timing behaviour of the source at different luminosity states, is on-going.

Acknowledgements. D.K. thanks Konstantin Postnov for useful suggestions. We thank the referee Dr. Lucien Kuiper for his comments and corrections which improved clearness and readibility of the paper. We wish to thank the Swift PI, Neil Gehrels, and the Staff of the Swift Missions Operation Center as well as the INTEGRAL Mission Scientist, C. Winkler, and the ESA ISOC personnel for their patient help in scheduling the simultaneous observations of this Target of Opportunity Program. This research is supported by the German Space Agency (DLR) under contracts Nos. 50 OG 9601 and 50 OG 0501.

\section{References}

Barthelmy, S. D., Barbier, L. M., Cummings, J. R., et al. 2005, Space Sci. Rev., 120, 143

Burrows, D. N., Hill, J. E., Nousek, J. A., et al. 2005, Space Sci. Rev., 120, 165 Coe, M. J. 2000, The Be Phenomenon in Early-Type Stars, ed. M. A. Smith, H. F. Henrichs, \& J. Fabregat, in IAU Colloq. 175, ASP Conf. Ser., 214, 656 Coe, M. J., Payne, B. J., Longmore, A., \& Hanson, C. G. 1988, MNRAS, 232, 865

Corbet, R. H. D., \& Levine, A. M. 2006, The Astronomer's Telegram, 843 Courvoisier, T. J.-L., Walter, R., Beckmann, V., et al. 2003, A\&A, 411, L53 Ferrigno, C., Segreto, A., Santangelo, A., et al. 2007, A\&A, 462, 995 Gehrels, N., Chincarini, G., Giommi, P., et al. 2004, ApJ, 611, 1005

Ghosh, P., \& Lamb, F. K. 1979, ApJ, 234, 296

Krimm, H., Barthelmy, S., Gehrels, N., et al. 2006, The Astronomer's Telegram 861

Lund, N., Budtz-Jørgensen, C., Westergaard, N. J., et al. 2003, A\&A, 411, L231 McCollough, M. L., Turler, M., Willis, D., \& Shaw, S. E. 2006, The Astronomer's Telegram 868

Parmar, A. N., White, N. E., Stella, L., Izzo, C., \& Ferri, P. 1989, ApJ, 338, 359 Pringle, J. E., \& Rees, M. J. 1972, A\&A, 21, 1

Reig, P., \& Coe, M. J. 1999, MNRAS, 302, 700

Ubertini, P., Lebrun, F., Di Cocco, G., et al. 2003, A\&A, 411, L131

Wilson, C. A., \& Finger, M. H. 2006, The Astronomer's Telegram 877

Wilson, C. A., Finger, M. H., Coe, M. J., Laycock, S., \& Fabregat, J. 2002, ApJ, 570,287

Wilson, C. A., Fabregat, J., \& Coburn, W. 2005, ApJ, 620, L99

Winkler, C., Courvoisier, T. J.-L., Di Cocco, G., et al. 2003, A\&A, 411, L1 